\title{
Conexões
}

\section{Incidência do flow-feeling e suas dimensões em praticantes de mountain bike}

\author{
Nadyne Nascimento dos Santos ${ }^{1}$ \\ Jadson Geovane de Brito Oliveira ${ }^{1}$ \\ Daniel Alvarez Pires ${ }^{1}$
}

\section{RESUMO}

As atividades físicas de aventura na natureza geram satisfação para seus praticantes e tal satisfação pode ser explicada com base na teoria do flow-feeling, a qual consiste em um estado mental no qual as pessoas parecem fluir, quando mostram um esforço produtivo e motivado. $\mathrm{O}$ presente estudo, do tipo qualitativo, objetiva investigar a incidência do flow-feeling e suas dimensões na prática do mountain biking. Para tal, dispomos de uma amostra composta por dez sujeitos, todos do sexo masculino e praticantes ativos. Os dados foram coletados por meio de entrevista semiestruturada. A análise dos dados foi feita a partir do método de análise de conteúdo. Foram avaliadas as Unidades de Contexto das respostas de cada praticante e as Unidades de Registro de cada dimensão do flow-feeling. Foram identificadas sete das oito dimensões nos relatos dos sujeitos. Concluiu-se que o flow-feeling se faz presente no mountain biking, com a ausência de apenas uma das dimensões - perda da noção do tempo.

Palavras Chave: Atividade motora. Natureza. Motivação.

\footnotetext{
${ }^{1}$ Universidade Federal do Pará Submetido em : 01 nov. 2016 Aceito em: 07 mar. 2018 Contato: danielpires@ufpa.br 


\section{Flow-feeling the implications and dimensions in mountain bike practitioners}

\section{Abstract}

Physical activities of adventure in nature generate satisfaction for its practitioners and such satisfaction can be explained based on the flow-feeling theory, which consists of a mental state in which people seem to flow when they show a productive and motivated effort. This study aims, the qualitative, to investigate the incidence of flow-feeling and its dimensions in the practice of mountain biking. To this end, we have a sample of ten subjects, all male and active practitioners. Data were collected through semi-structured interview. Data analysis was made from the content analysis method. The Context Units from the responses of each participant were evaluated, as well as the Registration Units of each dimension of the flow-feeling. Seven of the eight flow dimensions were identified in the reports of the subjects. I concluded that the flow-feeling is present in mountain biking, with the absence of only one of the dimensions loss of sense of time.

Keywords: Motor activity. Nature. Motivation.

\section{Flow-sintiendo la implicaciones $y$ dimensiones en profesionales bicicleta de montaña}

\section{RESUMEN}

Las actividades físicas de aventura en la naturaleza generan la satisfacción de sus practicantes y tal satisfacción se puede explicar en base a la teoría del flujo-sentimiento, que consiste en un estado mental en el que las personas parecen fluir cuando muestran un esfuerzo productivo y motivado. Este estudio, cualitativo, de tipo objetivo de investigar la incidencia de flujosentimiento y sus dimensiones en la práctica del ciclismo de montaña. Con este fin, tenemos una muestra de diez sujetos, todos los practicantes masculinos y activos. Los datos fueron recolectados a través de entrevista semiestructurada. El análisis de datos se realizó a partir del método de análisis de contenido. Las unidades de contexto se evaluaron las respuestas de cada participante y las unidades de registro de cada dimensión del flujo-sentimiento. Identificaron siete de las ocho dimensiones en los informes de los sujetos. Se concluyó que el flujo-sensación está presente en bicicleta de montaña, con la ausencia de uno solo de las dimensiones - pérdida del sentido del tiempo.

Palabras Clave: Actividad motora. Naturaleza. Motivación. 


\section{INTRODUÇÃO}

A prática de atividades físicas de aventura na natureza vem crescendo e se destacando entre os debates literários por constituírem uma questão que, segundo (LAVOURA; SCHWARTZ; MACHADO, 2008), transporta várias possibilidades investigativas no que diz respeito às práticas do contexto do lazer atual. Tais atividades como canoagem, pára-quedismo, mountain biking entre outras, podem proporcionar as mais diversas experiências esportivas aos praticantes.

A busca por atividades físicas de aventura na natureza pode estar relacionada ao contexto vivenciado na sociedade, ou seja, em um mundo globalizado e individualista, as pessoas percebem e passam a conviver com o risco que o mesmo traz. São formados grupos de acordo com a compatibilidade de seus interesses por essas práticas, explicitando emoções, sentidos, simbolismo e dimensões culturais. Tais grupos buscam o sentimento de superação dos seus próprios limites( BAHIA; SAMPAIO, 2008).

Uma das características principais da busca por essas atividades é a fuga da vida cotidiana e do estresse da vida urbana, a qual se reflete na procura pelo elemento "exótico" nas viagens à natureza. Indo ao encontro dos preceitos deste mundo globalizado, busca-se uma identidade própria, ou seja, busca-se o "ser diferente" canalizando assim motivações para vivenciar atividades na natureza (BAHIA; SAMPAIO, 2008).

O mountain biking (MTB) consiste em uma modalidade do ciclismo em que o objetivo é transpor percursos com várias irregularidades e obstáculos. Por se tratar de uma atividade física de aventura na natureza, pode ser caracterizado pelo alto risco em sua prática, assim como pela capacidade de proporcionar sensações e sentimentos de prazer, descanso, vertigem e risco. Uma de suas características mais marcantes é a superação de imprevisibilidades, tendo em vista que o objetivo desta atividade é vencer seus próprios limites pedalando sobre diversos tipos de terrenos com variadas alterações climáticas, os quais podem interferir na realização eficaz da atividade (LAVOURA; SCHWARTZ; MACHADO, 2008).

Para a prática do MTB existe uma bicicleta específica, a qual se diferencia das bicicletas de estrada em vários aspectos como: pneus mais largos para melhor absorverem os impactos; amortecedores que permitem um maior controle da bicicleta; quadros reforçados e mais resistentes; guidão que pode possuir vários formatos e estes devem se adequar às necessidades do ciclista; aros mais reforçados e pesados para evitar deformações nas ultrapassagens de obstáculos; relações de marchas que também devem se adequar às necessidades dos ciclistas entre outros aspectos.

De acordo com Miranda e Bara Filho (2008), as atividades de risco geram satisfação para seus praticantes e tal satisfação pode ser explicada com base na teoria do 
flow-feeling proposta por (CSIKSZENTMIHALYI, 2009). Tal teoria pode ser explicada como um "estado mental no qual as pessoas parecem fluir, quando mostram um esforço produtivo e motivado, associado às várias emoções relacionadas a comportamentos positivos e funcionais" 3 . Com isso, temos os praticantes entrando no estado ótimo da vivência, envolvendo-se com a atividade a ponto de não se perceberem separados da mesma - os indivíduos e as atividades se tornam únicos (MIRANDA; BARA FILHO, 2008).

A teoria do flow-feeling possui dimensões, independentemente da atividade, conhecidas como componentes do prazer. Tais componentes são: equilíbrio desafiohabilidade; objetivos claros e feedback imediato; perda da autoconsciência; concentração intensa na tarefa e fusão entre ação e atenção; perda da noção de tempo; controle absoluto das ações; alegria espontânea e experiência intrinsecamente compensadora; experiência autotélica( MIRANDA; BARA FILHO, 2008).

O fluxo exige um equilíbrio dinâmico entre desafio e habilidade, uma vez que os mesmos devem se corresponder e ultrapassar os níveis médios pessoais para que se consiga alcançar o estado mental ótimo(MASSARELLA; WINTERSTEIN, 2009). Portanto, precisa-se equilibrar a relação desafio-habilidade, de forma que haja proporção entre o grau de dificuldade da tarefa e o investimento de energia psicofísica disponível para a realização da mesma. É necessário também possuir objetivos claros e obter feedback imediato sobre suas ações, ou seja, deve-se desenvolver a percepção de que está apto para lidar com desafios iminentes, para que se possa chegar ao flow( MIRANDA; BARA FILHO, 2008).

De acordo com Oliveira (2009), o estado de fluidez gera um sentimento de união do indivíduo com o ambiente, seja este uma montanha ou carro de corrida, por exemplo. Esse sentimento de união constitui a perda da autoconsciência, ou seja, o praticante sente-se tão absorvido pela atividade que não sobra espaço para desperdício de atenção e a preocupação com o self - entidade que decide o que fazer com a energia psíquica gerada pelo sistema nervoso - desaparece. O estado de flow também pode acontecer quando há concentração intensa na tarefa e fusão entre ação e atenção. Esta dimensão muito se assemelha à anterior - perda da autoconsciência -, uma vez que ambas precisam de uma concentração completa na atividade. Essa concentração intensa na tarefa só é possível se a mente do praticante estiver livre de pensamentos irrelevantes e a fusão entre ação e atenção ocorre quando os processos psíquicos se fundem com os físicos, causando uma ausência de esforços na realização das ações (MIRANDA; BARA FILHO, 2008).

Quando se flui perde-se a noção de tempo, ou seja, o praticante se sente tão envolvido pela atividade que o relógio acaba sendo naturalmente esquecido, é como se ele estivesse em outra dimensão, onde o tempo não passa ou passa em questão de segundos. Três praticantes de corrida de rua relataram ter essa sensação de que o tempo passou muito mais rápido do que realmente havia ocorrido (MASSARELLA; 
WINTERSTEIN, 2009). O fluir também gera o controle absoluto das ações, o praticante percebe que possui controle total sobre seu corpo e se torna confiante para realizar determinada tarefa. É por conta dessa percepção de controle absoluto que certas atividades consideradas de risco como o MTB, por exemplo, provocam prazer aos praticantes, tendo em vista que os esportes de risco são os melhores modelos para exemplificar esta dimensão (MIRANDA; BARA FILHO, 2008).

Para que se alcance o estado de flow o praticante não deve visar recompensas externas à atividade, pois tudo aquilo que é externo é compreendido como consequência natural e não precisa ocupar sua mente ${ }^{5}$. Exercer a atividade já é a própria recompensa para o indivíduo (MASSARELLA; WINTERSTEIN, 2009). Portanto, o simples fato de poder realizar a atividade já lhe causa uma alegria espontânea e ele passa por uma experiência intrinsecamente compensadora. A essa experiência compensadora, na qual o fim da atividade está em si mesmo, dá-se o nome de experiência autotélica, que consiste no resultado final do flow-feeling e é o elemento fundamental de toda a experiência (MIRANDA; BARA FILHO, 2008).

As dimensões equilíbrio entre desafio e habilidade, metas claras e retorno (feedback) e concentração total na atividade e no momento são os elementos necessários para que ocorra oflow. Perda da autoconsciência e da noção de tempo, controle absoluto das ações, alegria espontânea e experiência intrinsecamente compensadora e experiência autotélica são interpretadas como consequências ou percepções da ocorrência do flowfeeling. Essa divisão, segundo Massarella e Winterstein (2009), visa facilitar a compreensão do elemento analisado (flow-feeling), assim como facilita a análise de dados no estudo.

Além das dimensões explicitadas acima, Weinberg e Gould (2008) citam outro elemento chamado movimento sem esforço. $\mathrm{O}$ mesmo refere-se a um estado em que o praticante está vivenciando a atividade tão profundamente que parece não estar se esforçando, ou seja, como se suas ações acontecessem automaticamente.

A teoria do flow-feeling, de acordo com Weinberg e Gould (2008), concebe uma importantíssima inovação nas investigações a respeito de motivação intrínseca. Determinadas atividades proporcionam a capacidade de gerar experiências máximas e envolvem elevado potencial de motivação intrínseca (MIRANDA; BARRA FILHO, 2008). Segundo Massarella e Winterstein (2009), quando a motivação é ntrínseca a pessoa não visa recompensas externas, ou seja, as mesmas podem surgir, mas não representam o foco da prática e por se tratar de um fenômeno que envolve apenas a pessoa e o prazer pela atividade, esse tipo de motivação pode levar ao flow.

Compreende-se que esta área é emergente e possui relevância para as produções científicas, uma vez que existe a necessidade de investigação para identificar por que as atividades físicas de aventura na natureza constituem grande fonte de motivação intrínseca. Portanto, esta investigação trará novas (re) significações às práticas de Conexões: Educ. Fís., Esporte e Saúde, Campinas: SP, v. 15, n. 4, p. 452-464, out./dez. 2017. ISSN: 1980-9030 
atividades físicas de aventura na natureza. Diante do exposto, o presente estudo objetiva investigar a incidência doflow-feeling e suas dimensões em praticantes de MTB.

\section{MÉTodo - Tipo De Estudo}

Esta pesquisa é qualitativa e este tipo de investigação, segundo Thomas; Nelson e Silverman (2007) consiste em um método de estudo o qual segue o método científico de solução de problemas. Tal método é constituído por um campo de complexos estudos que ultrapassa as fronteiras disciplinares e se adéqua aos estudos de problemas de conhecimento científico, em variados campos da atividade humana (GOELLNER, 2010).

As investigações com métodos de caráter qualitativo têm se destacado em diversas pesquisas relacionadas à psicologia do esporte e do exercício pelo fato de proporcionar uma determinada abrangência de informações, sendo consideradas extremamente úteis na identificação de novas variáveis em áreas pouco exploradas ou no entendimento de aspectos emocionais e cognitivos dos sujeitos (BENTES, 2014; DIAS; CRUZ; FONSECA, 2009). Baseando-se neste método de investigação, o presente estudo almeja dispor de dados aprofundados que levem em consideração as percepções subjetivas dos sujeitos investigados sobre a manifestação das dimensões do flow-feeling.

\section{Amostra e Cuidado Ético}

Foram selecionados dez ciclistas praticantes de MTB, todos participantes de um grupo chamado "Grupo Trilheiros de Castanhal (GTC)". O GTC possui onze anos de existência e é composto por pessoas que se dispõem a pedalar três vezes por semana às terças e quintas à noite e uma vez no fim de semana - fazendo trilhas dentro da cidade e em suas redondezas.

Como critério de inclusão, todos deveriam ser do sexo masculino e os mesmos deveriam estar ativos no momento da seleção (praticar pelo menos três vezes por semana), possuir mais de 18 anos e pelo menos um ano de prática. O número da amostra foi definido de acordo com os critérios de inclusão e com a disponibilidade dos membros do grupo selecionado para a pesquisa. $O$ esporte não foi analisado sob perspectiva de competição, e sim como prática de atividade física de aventura na natureza vivenciada em momentos reservados para tal. O estudo obedeceu à Resolução 466/2012, que versa sobre os procedimentos éticos envolvendo seres humanos. Portanto, ao aceitarem participar da pesquisa, os participantes assinaram um Termo de 
Consentimento Livre e Esclarecido (TCLE) (APÊNDICE A) autorizando a utilização dos dados.

\section{INSTRUMENTOS}

Os sujeitos preencheram uma ficha sociodemográfica (APÊNDICE B) fornecendo informações sobre sua relação com o MTB. A análise dos dados foi feita a partir das respostas dos entrevistados às seguintes perguntas adaptado de estudo feito por Massarella e Winterstein (2009) (APÊNDICE C): quando você começou a se interessar pelo MTB e por quê? Por que você pratica o MTB atualmente? Quais os sentimentos mais comuns quando você está praticando o MTB? Quais os pensamentos mais comuns quando você está praticando o MTB? Relate uma experiência com o MTB que tenha sido marcante para você; O MTB para mim é... Deixe uma mensagem para as pessoas sobre o MTB.

\section{Procedimentos}

A recolha dos dados foi agendada previamente com os participantes e aconteceu em uma das salas de aula da Universidade Federal do Pará/Campus Universitário de Castanhal, ambiente isolado e livre de ruídos. As entrevistas foram aplicadas individualmente.

\section{Análise de Dados}

Utilizou-se o método de análise de conteúdo de Bardin para análise dos dados. Essa técnica tem sido empregada na literatura em Psicologia do Esporte e do Exercício (MASSARELLA; WINTERSTEIN, 2008; BENTES, 2014; SANTIAGO, 2016), e a mesma é composta por três fases: fase de organização, em que as ideias iniciais são estruturadas; fase de exploração do material, na qual ocorre a aplicação sistemática das decisões tomadas; e fase de tratamento dos efeitos obtidos e interpretação, em que os resultados brutos são tratados de maneira a serem significativos e válidos (BARDIN, 2011) ${ }^{4}$. Foi realizada codificação, de forma a garantir o anonimato dos ciclistas participantes. Elaborou-se um código alfanumérico formado pelas letras MTB com os números de 1 a 10, de acordo com a ordem das entrevistas (BARDIN, 2014; SANTIAGO, 2016). 


\section{Resultados}

A categorização é uma forma de organização da análise proposta por (BARDIN, 2011) que consiste em organizar os dados a priori, ou seja, os documentos adquiridos por meio da coleta (entrevista) são distribuídos da maneira mais coerente a cada um dos elementos do fenômeno que se deseja investigar - referenciais teóricos já fornecidos pela literatura. A análise de conteúdo identificou a presença de sete das oito dimensões do flow-feeling investigadas no presente estudo (QUADRO 1). Os dados analisados passaram por um processo de categorização em Unidades de Registro e Unidades de Contexto. As Unidades de Registro representam as dimensões do elemento analisado e as unidades de contexto representam a incidência de cada dimensão por meio dos discursos dos sujeitos entrevistados.

Quadro 1- Dimensões do flow-feeling identificadas em praticantes de MTB

\begin{tabular}{|c|c|}
\hline Unidades de registro & Unidades de contexto \\
\hline Equilíbrio desafio-habilidade & $\begin{array}{l}\text { MTB3: }[\ldots] \text { uma prova de }[\ldots] \text { cento e dez quilômetros }[\ldots] \\
\text { fomos pra lá confiantes de que estávamos aptos a cumprir a } \\
\text { prova. MTB4: }[\ldots] \text { gente pedala trezentos quilômetro }[\ldots] \text { é } \\
\text { um desafio pedalar trezentos quilômetro. }\end{array}$ \\
\hline Objetivos claros e feedback imediato & $\begin{array}{l}\text { MTB7: [...] eu via que tinha que ter superação, não se } \\
\text { tratava de, de depender de um grupo pra você ir mais longe, } \\
\text { era algo seu. } \\
\text { MTB9: [...] um sentimento muito de desafio [...] e de poxa } \\
\text { eu posso fazer aquilo, eu posso andar naquela velocidade, } \\
\text { naquele ritmo. }\end{array}$ \\
\hline Perda da autoconsciência & $\begin{array}{l}\text { MTB3: [...] quando eu estou praticando o mountain biking } \\
\text { é o momento onde eu me abstraio até do próprio mundo. } \\
\text { MTB10: [...] É um sentimento de liberdade, de poder } \\
\text { esquecer de tudo. }\end{array}$ \\
\hline $\begin{array}{l}\text { Concentração intensa na tarefa e fusão entre } \\
\text { ação e atenção }\end{array}$ & $\begin{array}{l}\text { MTB1: [...] o mountain biking ele te dá uma leveza no } \\
\text { corpo. } \\
\text { MTB3: [...] Eu só to curtindo aquele momento, então na } \\
\text { verdade eu não lembro de problemas, não lembro de } \\
\text { família ali, não lembro de nada, eu só aproveito o } \\
\text { momento. }\end{array}$ \\
\hline Controle absoluto das ações & $\begin{array}{l}\text { MTB6: }[\ldots] \text { calma }[\ldots] \text { eu tenho muita calma }[\ldots] \\
\text { principalmente quando ta em um, em locais de riscos. } \\
\text { MTB8: }[\ldots] \text { pode até alongar se for prum lugar que, que dá } \\
\text { cem quilômetro por asfalto pode jogar cento e oitenta por, } \\
\text { por trilha, ramal que eu to dentro. }\end{array}$ \\
\hline $\begin{array}{l}\text { Alegria espontânea } \quad \mathrm{e} \\
\text { intrinsecamente compensadora }\end{array}$ & $\begin{array}{l}\text { MTB7: [...] E o ciclismo hoje e sempre foi um ciclismo que } \\
\text { você faz por você, o restante é consequência, ganhar } \\
\text { amigos, ganhar um grupo é consequiência. } \\
\text { MTB8: [...] E eu curto mountain biking porque é uma coisa } \\
\text { que dá prazer né [...] É uma felicidade e tanto! }\end{array}$ \\
\hline Experiência autotélica & $\begin{array}{l}\text { MTB7: [...] É... além de ser minha profissão, é o meu } \\
\text { esporte e é o meu hobby. [...] é... o meu meio de vida [...] } \\
\text { faz parte da minha vida literalmente, vinte e quatro horas. } \\
\text { MTB8: [...] Ah é tudo! Meu esporte, é meu hobby, é um } \\
\text { co... é uma paixão. É dumas paixão que eu tenho é o } \\
\text { mountain biking. }\end{array}$ \\
\hline
\end{tabular}


A dimensão perda da noção de tempo foi a única não identificada dentre as demais, uma vez que não surgiu em nenhum dos relatos verbais dos sujeitos entrevistados.

\section{Discuss ÃO}

O presente estudo de cunho qualitativo objetivou investigar a incidência do flowfeeling e suas dimensões na prática do MTB. O mesmo identificou a presença de sete das oito dimensões do elemento, abordadas dentro dos relatos verbais dos sujeitos.

A correspondência entre desafio e habilidade foi verificada nos relatos dos sujeitos MTB3 e MTB4 registrados nas unidades de contexto, em que os mesmos demonstraram percepção de possuírem certa aptidão pessoal para o MTB. Tal percepção, somada à percepção da capacidade de responder aos desafios, constituem um importante componente para a ocorrência do flow-feeling: equilíbrio desafio-habilidade. De acordo com (CSIKSZENTMIHALY, 1999), o estado de fluxo tende a ocorrer quando as habilidades da pessoa estão focadas na superação de um desafio que está no limiar da sua capacidade de domínio. Portanto, a relação entre essa percepção de competência e o flow-feeling é diretamente proporcional, ou seja, quando a percepção aumenta, torna-se mais fácil ao indivíduo alcançar um estado ótimo.

Foi possível identificar a dimensão objetivos claros e feedback imediato nos relatos dos sujeitos MTB7 e MTB9, nos quais os mesmos deixaram transparecer certa capacidade de alcançar seus objetivos por meio de um feedback preciso. Para se ter objetivos claros, é necessário receber um feedback do seu desempenho para assim poder alcançá-los e num estado de flow-feeling torna-se mais fácil obter esse retorno de forma clara e sem ambiguidades (CSIKSZENTMIHALY, 1999; SENA JUNIOR, 2012). Apesar de não ter sido abordado pelos participantes do presente estudo, é comum entre os ciclistas o monitoramento (feedback) para avaliar se os mesmos estão conseguindo atingir suas metas e para isso eles utilizam um instrumento que registra o tempo e a velocidade, assim como outras variáveis.

Os sujeitos MTB3 e MTB10 deixaram transparecer que quando pedalam se abstraem de tudo, "até do próprio mundo" (MTB3). Desta forma, o praticante se envolve tão profundamente com a atividade que acaba ocorrendo uma perda da autoconsciência, ou seja, o envolvimento é tão profundo que não sobra espaço para desperdício de atenção e a preocupação com o self desaparece (MASSARELLA; WINTERSTEIN, 2009). O flow-feeling é mais comumente relatado quando a pessoa está realizando sua atividade favorita e por se tratar de uma atividade física de aventura na natureza, o praticante deve de fato gostar do MTB para vivenciá-lo e assim, não se preocupar com situações externas à atividade. 
A dimensão concentração intensa na tarefa e fusão entre ação e atenção foi observada nos relatos dos sujeitos MTB1 e MTB3 nos quais se pode perceber com clareza uma ausência de esforços devida à fusão de processos físicos e psíquicos somada a uma intensa concentração. Quando se faz o que se gosta o flow-feeling é mais fácil de ser alcançado, pois a concentração na tarefa é completa e intensa e os processos físicos e psíquicos se fundem, considerando que a mente está livre de quaisquer pensamentos externos à atividade (MIRANDA JÚNIOR, 2012). Nas atividades físicas de aventura na natureza o risco torna-se um fator instigante, uma vez que as pessoas estão emocionalmente envolvidas com a atividade e esta, por se tratar de uma prática que pode oferecer diversos tipos de ameaças ao indivíduo, o mesmo precisa estar completamente concentrado no que faz.

Os sujeitos MTB6 e MTB8 deixaram transparecer em seus relatos uma determinada percepção de controle absoluto e autoconfiança para a realização da tarefa. De acordo com Miranda e Bara Filho (2008), determinadas atividades que oferecem perigo também geram satisfação aos seus praticantes e os esportes de risco são os melhores exemplos, pois os mesmos dão ênfase à dimensão controle absoluto das ações. Não é do sentimento de controle que o praticante gosta e sim de conseguir exercer o controle em situações difíceis (OLIVEIRA, 2013).

Todos os praticantes expressaram uma alegria e satisfação contagiantes pelo MTB em seus relatos, o que caracteriza a dimensão alegria espontânea e experiência intrinsecamente compensadora. Ao vivenciar uma atividade física de aventura na natureza, o praticante sente certo receio, por vezes, diante do inesperado. Em contrapartida, a capacidade de transpor um obstáculo proporciona uma alegria espontânea, o que torna a atividade intrinsecamente compensadora, além do fato de tais vivências consistirem em tarefas intrinsecamente motivadoras, pois as mesmas proporcionam aos seus praticantes experiências gratificantes e devem ser realizadas por vontade própria (VIEIRA, 2011; SILVA; FREITAS, 2010; STRASSBURGER; MACKE, 2012).

O destaque de uma atividade autotélica é que seu praticante foca sua atenção na própria tarefa e não em futuras recompensas e essa dimensão é muito bem exemplificada nos relatos dos sujeitos MTB7 e MTB8. A experiência autotélica consiste no resultado final do flow-feeling. Ela é o elemento fundamental de toda a experiência e ao vivenciá-la, o praticante percebe que o fim da atividade está em si mesmo, ou seja, não importa o que vem depois da prática, o simples fato de poder realizá-la já é a própria recompensa (CSKSZENTMIHALYI,1999). O que caracteriza uma experiência autotélica é a satisfação de se fazer o que gosta sem visar lucros(SENA JÚNIOR, 2012) e isso foi percebido nos relatos dos sujeitos, uma vez que os mesmos relataram praticar o MTB em busca de uma simples satisfação pessoal, ou seja, motivados intrinsecamente. 
A única dimensão não identificada nas análises foi a perda da noção do tempo, o que também ocorreu em estudos realizados por (MIRANDA JÚNIOR, 2012; GOMES, 2014; OLIVEIRA; MIRANDA, 2015). Segundo Miranda Júnior (2012), a perda da noção do tempo é uma das características menos frequentes no tênis, assim como em outros esportes. Portanto, percebeu-se que esta é a dimensão menos central para os sujeitos desta amostra e isso pode ser explicado pelo fato de que existem atividades nas quais o tempo é essencial para os desafios da tarefa, ou seja, a capacidade de controlar o tempo deve ser mantida para se obter êxito e o esporte abordado se encaixa nessa característica, uma vez que os praticantes precisam ter esse controle do tempo para melhorar seu desempenho.

Este estudo apresentou limitações que não possibilitam a generalização dos resultados, como a quantidade de amostra, uma vez que esta foi relativamente escassa para uma pesquisa mais abrangente e o fato de o grupo investigado dispor de apenas uma mulher em sua composição e esta não ter sido incluída na investigação.

\section{Conclusão}

Os participantes do estudo revelaram a presença da maioria das dimensões do flow-feeling durante suas práticas, com exceção de apenas uma - perda da noção do tempo - a qual também é menos frequente em outros esportes. A incidência deste fenômeno no MTB reforça um comportamento positivo em relação à atividade, uma vez que pode contribuir para a permanência do sujeito no esporte - por se tratar de uma atividade intrinsecamente motivadora -, assim como a paixão demonstrada por todos os participantes em relação ao MTB.

São necessários mais estudos que relacionem o flow-feeling aos esportes para que assim possamos compreender com mais precisão este fenômeno tão inconscientemente vivenciado na maioria das atividades, porém relativamente pouco investigado num contexto nacional. Portanto, faz-se necessária a realização de mais pesquisas, especificamente relacionando o MTB com o flow-feeling, utilizando um número maior de participantes que inclua mulheres na amostra, uma vez que, por se tratar de uma atividade de aventura na natureza que oferece risco ao seu praticante, o mesmo deve ser vivenciado a partir de uma motivação intrínseca, ou seja, a pessoa precisa gostar para praticar. 


\section{REFERÊNCIAS}

BAHIA, Mirleide Chaar; SAMPAIO, Tânia Mara Vieira. Lazer-meio ambiente: em busca das atitudes vivenciadas nos esportes de aventura. Revista Brasileira de Ciências do Esporte, Campinas, v. 28, n. 3, p. 173-189, 2007.

BARDIN, Laurence. Análise de conteúdo. São Paulo: Edições 70, 2011. 279p.

BENTES, Lívia Maria Neves. Percepção de estresse no contexto da ginástica rítmica: um estudo com jovens ginastas. 2014. 75f. (Licenciatura em Educação Física) Universidade Federal do Pará, Castanhal, 2014.

CSKSZENTMIHALYI, Mihaly. A descoberta do fluxo: a psicologia do envolvimento com a vida cotidiana. Rio Claro: Rocco, 1999. 166p.

DIAS, Cláudia; CRUZ, José Fernando; FONSECA, António Manuel. Emoções, strees, ansiedade e coping: estudo qualitativo com atletas de elite. Revista Portuguesa de Ciência do Desporto, Porto, v. 9, n. 1, p. 9-23, 2009.

GOELLNER, Silvana Vilodre et al. Pesquisa qualitativa na educação física brasileira: marco teórico e modos de usar. Revista da Educação Física/UEM, Maringá, v. 21, n. 3, p. 381-410, 2010.

GOMES, Simone Salvador et al. O fluxo no voleibol: relação com a motivação, autoeficácia, habilidade percebida e orientação às metas. Revista de Educação Física/UEM, Maringá, v. 23, n. 3, p. 379-387, 2012.

GOMES, Simone Salvador. Evidências psicométricas da escala de predisposição ao fluxo (DFS-2) para brasileiros. 2014. 132 f. (Doutorado em Educação Física) Universidade São Judas Tadeu, São Paulo, 2014.

LAVOURA, Tiago Nicola; SCHWARTZ, Gisele Maria; MACHADO, Afonso Antônio. Aspectos emocionais da prática de atividades de aventura na natureza: a (re) educação dos sentidos. Revista Brasileira de Educação Física e Esportes, São Paulo, v. 22, n. 2, p. 119-27, 2008.

MASSARELLA, Fábio Luiz; WINTERSTEIN, Pedro José. A motivação intrínseca e o estado mental flow em corredores de rua. Movimento, Porto Alegre, v. 15, n. 2, p. 45-68, 2009.

MIRANDA, Renato; BARA FILHO, Maurício. Construindo um atleta vencedor: uma abordagem psicofísica do esporte. Porto Alegre: Artmed, 2008. 
MIRANDA JÚNIOR, Márcio Vidigal et al. Análise do flow-feeling no tênis. Revista de Educação Física/UEM, Juiz de Fora, v. 23, n. 4, p. 607-615, 2012.

OLIVEIRA, Helder Zimmermann. Flow-Feeling no esporte: uma revisão bibliográfica. 2009. 30f. (Bacharelado em Educação Física) - Faculdade de Educação Física e Desporto, Universidade Federal de Juiz de Fora, Juiz de Fora, Juiz de Fora, 2009.

Motivação e flow-feeling em jovens jogadores do basquetebol. 2013. 122f. (Mestrado em Educação Física) - Universidade Federal de Juiz de Fora, Juiz de Fora, 2013.

.; MIRANDA, Renato. O estado de flow no basquetebol em categorias de base: uma análise da interferência da idade e do tempo de prática. Revista de Educação Física/UEM, Maringá, v. 26, n. 1, p. 89-96, 2015.

SANTIAGO, Marisa Lúcia Mello et al. Síndrome de burnout em treinadores brasileiros de voleibol de alto rendimento. Revista de Psicología Del Deporte, Belo Horizonte, v. 25, n. 2, p. 35-41, 2016.

SENA JÚNIOR, Antonio Walter. Motivação e flow-feeling na corrida de rua. 2012. 114 f (Mestrado em Educação Física) - Universidade Federal de Juiz de Fora, Juiz de Fora, 2012.

SILVA, Priscilla Pinto Costa; FREITAS, Clara Maria Silvestre Monteiro. Emoções e riscos nas práticas na natureza: uma revisão sistemática. Motriz, Rio Claro, v. 16, n. 1, p. 221-230, 2010.

STRASSBURGER, Nândri Cândida; MACKE, Janaina. Dimensões de análise da experiência do flow no turismo de aventura: rafting em Nova Roma do Sul (RS/Brasil). Revista Turismo Visão e Ação, Caxias do Sul, v. 14, n. 2, p. 150-163, 2012.

THOMAS, Jerry R.; NELSON, Jack K.; SILVERMAN, Stephen J. Métodos de pesquisa em atividade física. 5. ed. Porto Alegre: Artmed, 2007. 400p.

VIEIRA, Lenamar Fiorese et al. Estado de fluxo em praticantes de escalada e skate downhill. Motriz, Rio Claro, v. 17, n. 4, p. 591-599, 2011.

WEINBERG, Robert S.; GOULD, Daniel. Fundamentos da psicologia do esporte e do exercício. 4. ed. Porto Alegre: Artmed, 2008. 624p. 

ERROR: syntaxerror

OFFENDING COMMAND: --nostringval--

\section{STACK :}

/ Title

()

/ Subject

(D:20180405152556-03'00')

/ModDate

()

/Keywords

(PDFCreator Version 0.9.5)

/Creator

(D:20180405152556-03'00')

/CreationDate

(dulce)

/Author

-mark- 